\title{
Novel synthetic route for growth of gold nanorods via semiconductor procedure
}

\author{
Noha Elhalawany ${ }^{1 *}$, Brian Enders ${ }^{2}$, Ersin Bahceci ${ }^{3}$, Munir Nayfeh $^{2}$ \\ ${ }^{1}$ Polymers and pigments dept., National Research Center, Cairo, Egypt \\ ${ }^{2}$ Physics dept., University of Illinois at Urbana Champaign, USA \\ ${ }^{3}$ Dept. of Metallurgical and Materials Engineering Iskenderun Teknik University, Turkey \\ *Correspondence: elhalawany1933@gmail.com
}

\begin{abstract}
We represent here a novel facile synthesis type route based on semiconductor procedure for growth of gold nanorods GNRs using infinitesimal silicon nanoparticles USSiN. The reaction takes place immediately upon mixing monodispersed hydrogen terminated USSiN of $2.9 \mathrm{~nm}$ diameter with auric acid $\mathrm{HAuCl}_{4}$ in presence and in absence of an emulsifier. The resulting colloids have been characterized via scanning electron microscope SEM, Energy dispersive spectrometry EDS and optical microscope OM. Photo-luminesence (PL) measurements have been also carried out. Our results show formation of gold nanorods GNRs, gold nanoplates GNPs, gold nanospheres GNSs and filaments. The formed GNRs have near uniform length of $1.5 \mu \mathrm{m}$ and diameter of $300 \mathrm{~nm}$ ( 5 aspect ratio). The results are consistent with a seedless process in which the H-terminated silicon nanoparticles act as either the reducing as well as the directional growth agent, eliminating the need for toxic cetyl-trimethyl-ammonium bromide CTAB or, which is typically used as the directional growth agent.
\end{abstract}

Keywords: infinitesimal silicon nanoparticles; gold nanorods; directional growth agent

Received: Mar 15, 2019 Accepted: May 13, 2019 Online: May 27, 2019

\section{Introduction}

There has been a great interest in exploiting nanomaterials due to their countless uses in various applications such as energy storing systems, solar cells, chemical catalysis and innovative cancer therapies ${ }^{[1-4]}$. Gold nanoparticles GNPs are one of the most important class of nanomaterials due to its ease of preparation and functionalization ${ }^{[4]}$. In particular, gold nanorods (GNRs) which have a broad tunable aspect-ratio dependent longitudinal surface plasmon resonance (LSPR), are mainly interesting due to their unique optical properties ${ }^{[4-6]}$. Therefore, GNRDS have been used in various applications especially biomedical applications such as a bio-sensing or biomedical device ${ }^{[2,7-9]}$ model systems for molecular imaging and targeted therapy ${ }^{[10]}$ and drug delivery systems ${ }^{[1]}$.

Up to now a number of studies for the preparation of gold nanorods have been reported involving a porous aluminum oxide membrane ${ }^{[12]}$, an electrochemical method with surfactant molecules as directing agents ${ }^{[13]}$, photochemical ${ }^{[14,15]}$, sonochemical ${ }^{[16]}$, and radiation-chemical ${ }^{[17,18]}$ methods. The most used synthesis type route to obtain GNRs with a controlled size and shape is the seed-mediated growth method, which was initially developed by Obare et al. ${ }^{[19]}$, and Sau and Murphy ${ }^{[20]}$. Seed-mediated growth occurs through two steps or several pots: the first step is to prepare gold seeds of definite particle size, and the other steps are growth steps of the seeds in presence of cetyltrimethylammonium bromide $\mathrm{CTAB}$ as a growth directional agent, ascorbic acid as reducing agent and $\mathrm{AgNO}_{3}$ as size controlling agent. Recently, many modified seed-mediated growth methods have been reported by various researchers ${ }^{[6,21-26]}$.

However, there are two problems that limit the GNRs applications first, the presence of (CTAB) on the surface of GNRs which is highly toxic and second, the removal of CTAB may also result in uncontrollable aggregation of GNRs ${ }^{[27]}$. The mechanism of rod formation is still complex and not very clear. Also, using the same experimental conditions did not achieve exactly the same size and shape of GNRs ${ }^{[28]}$.

\footnotetext{
Copyright (C) 2019 Noha Elhalawany et al. This is an Open Access article distributed under the terms of the Creative Commons AttributionNonCommercial 4.0 International License (http://creativecommons.org/licenses/by-nc/4.0/), permitting all non-commercial use, distribution, and reproduction in any medium, provided the original work is properly cited.
} 
In present work, we show for the first time that we can grow gold nanorods, plates, spheres and filaments from gold ions using USSiN seeds as both reducing and growth directional agent eliminating the use of toxic CTAB or metalbased reducers. A plausible mechanism for rod formation is also proposed.

\section{Material and Methods}

\subsection{Materials}

All chemicals used are products of Sigma-Aldrich, USA.

\subsection{Silicon nanoparticles formation:}

Silicon nanoparticles, $2.9 \mathrm{~nm}$ in diameter have been prepared by Nayfeh et al. ${ }^{[29]}$ from Si wafers by chemical etching in hydrofluoric acid/ hydrogen perroxide $\mathrm{HF} / \mathrm{H}_{2} \mathrm{O}_{2}$ solution using an electric current or hexa-chloroplatinic acid catalyst. The nanoparticles are H-terminated (Si-H) and have band gab of a $2.060 \mathrm{eV}{ }^{[30]}$. Nanoparticle suspensions are prepared in isopropyl alcohol (IPA). Monte Carlo simulation of the Si nanoparticle suggests a filled fullerene structure of $\mathrm{Si}_{29} \mathrm{H}_{24}$, in which a central core silicon atom and four other silicon atoms are arranged in a tetrahedral coordination and the 24 remaining silicon atoms undergo a H-terminated bulk-like $(2 \times 1)$ reconstruction of dimer pairs on $(001)$ facets (6 reconstructed surface dimers) ${ }^{[31]}$. The structure of the silicon nanoparticle is represented in Figure 1.

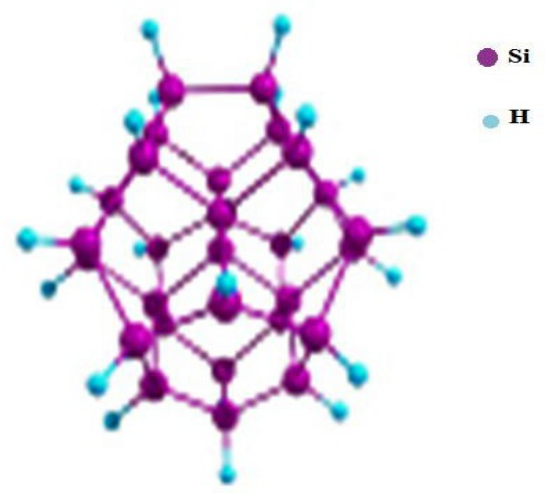

Figure 1. Ultrasmal silicon nanoparticle USSiN ${ }^{[31]}$.

\subsection{Formation of gold nanorods GNRs in absence of an emulsifier:}

We use a solution of $10 \mathrm{mM}$ of auric acid $\left(\mathrm{HAuCl}_{4}\right)$ as a source of gold ions. In the procedure, $0.5 \mathrm{ml}$ of $1.65 \mathrm{mM}$ auric acid solution is added to Si nanoparticles dispersed in IPA solution. A fast reaction occurs leading to change in color from yellow to pale pink color characteristic for gold nanoparticles.

\subsection{Formation of gold nanorods GNRs in presence of an emulsifier:}

$0.05 \mu \mathrm{L}$ dodecyl benzene sulfonic acid DBSA as an emulsifier has been added to silicon nanoparticles dispersion in isopropyl alcohol IPA, followed by adding $0.5 \mathrm{ml}$ of $1.65 \mathrm{mM}$ auric acid $\mathrm{HAuCl}_{4}$ solution under continuous vigorous stirring using high shearing effect homogenizer. The reaction takes place immediately giving rise to fast color change from yellow to pale pink color characteristic for gold nanoparticles.

\subsection{Characterization of GNRs}

\subsubsection{Scanning electron microscope (SEM):}

Samples have been measured using SEM JOEL 6060 LV, Japan at the MRL Lab, University of Illinois - Urbana Champaign (UIUC).

\subsubsection{Light microscope (LM):}

The samples have been examined by Mitutoyo Micro-scope, MRL fabrication facility, University of Illinois at Urbana Champaign (UIUC), USA.

\subsubsection{Photoluminescence spectroscopy}

A fiber-optic spectrometer (ocean optics, USB4000, USA) with excitation in the UV region is used to record the luminescence spectra of the prepared samples. 


\section{Results and discussion}

GNRDs have great potential for applications in display technologies because the reflectivity of the rods can be changed by changing their orientation with an applied electric field for; as well as in microelectromechanical systems (MEMS). Moreover, nanorods may find application in biomedical imaging, drug delivery and photothermal therapy due to unique surface plasmon resonance (SPR) ranging from visible to near infrared (NIR) region. The mechanism of formation of GNRDs has received concentrated activity in recent years yet it is not completely understood. However, it has now been demonstrated that the necessary ingredients for forming GNRs ${ }^{[20]}$ are (i) the presence of gold ions in solutions, (ii) a mild reducing agent, (iii) a shape-control or directing surfactant, which forms a rod shaped template that is filled with gold atoms (CTAB for example), otherwise, only spheres would form, and finally (iv) a protecting agent to prevent aggregation ( such as polyvinyl alcohol PVA and CTAB (plays this role) for example). In this paper, we report on a new facile synthesis type route in which we use monodispersed H-terminated USSiN of $2.9 \mathrm{~nm}$ diameter as both reducing as well as directional growth agent, eliminating the need for CTAB as a directional growth agent. Forming metal gold structures from gold ions requires a reducing agent. It is plausible that the silicon nanoparticles act as a reducer. Once gold ions are attracted to the silicon nanoparticles, they get reduced spontaneously. The overall neutral reduction reaction is as follows:

$$
\mathrm{HAuCl}_{4}+\mathrm{SiH}=\mathrm{Au}+2 \mathrm{HCl}+\mathrm{SiCl}_{2}
$$

After gold ions got reduced, the formed gold atoms diffuse in the particle, making an amorphous agglomerate of Au-Si, which act as a gold seed. More and more ions get reduced and diffuse to the gold seed, while pushing the silicon material outward in a near floating condition. This action is assembling into a gold nano structure, most likely a wire or rod configuration. The mechanism of Formation of GNRs is represented in Figure 2.

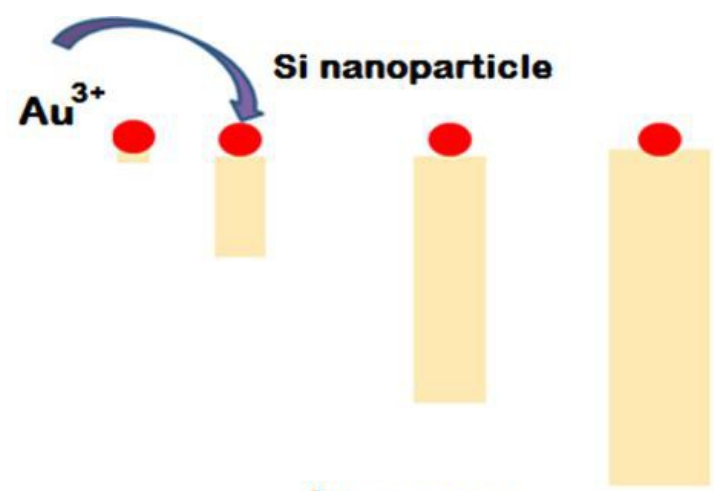

\section{Au nanorod}

Figure 2. Gold nanorod GNRs formation

\subsection{Characterization of GNRs}

\subsubsection{Scanning electron microscope SEM}

The resulting colloids have been characterized by SEM. A thin film was made by dispensing a certain volume of the formed colloid on device quality silicon wafer. Figure 3 gives a scanning electron microscope image of the colloid formed in presence of DBSA. Our results show formation of gold nanorods with near uniform length of $1.5 \mu \mathrm{m}$ and diameter of $300 \mathrm{~nm}$ (5 aspect ratio). One can see also some non-cylindrical GNRs. The figure also shows examples of close up of monodisoersed GNRDs and GNSs. The sizes of GNSs are in the range from 200-300 nm. The GNRDs lengths are in the range from 1.5 to $5 \mu \mathrm{m}$. The rods of length $1.5 \mu \mathrm{m}$ and diameter $300 \mathrm{~nm}$ (aspect ratio of 4-5) are more frequent.

Figure 4 shows SEM image for the colloidal dispersion prepared in absence of an emulsifier. The figure shows formation of massive assembly of gold filaments.

Figure 5 displays other nano structures that this procedure in absence of an emulsifier can yield, including gold nanoplates GNPs (squares). 
The gold-silicon (Au-Si) binary system belongs to the eutectic-alloy compounds, which exhibits lower melting temperatures and transform directly between solid and liquid ${ }^{[32]}$. This enables wafer-bonding, device connection and growth of Si nanowires using nanogold seeds.
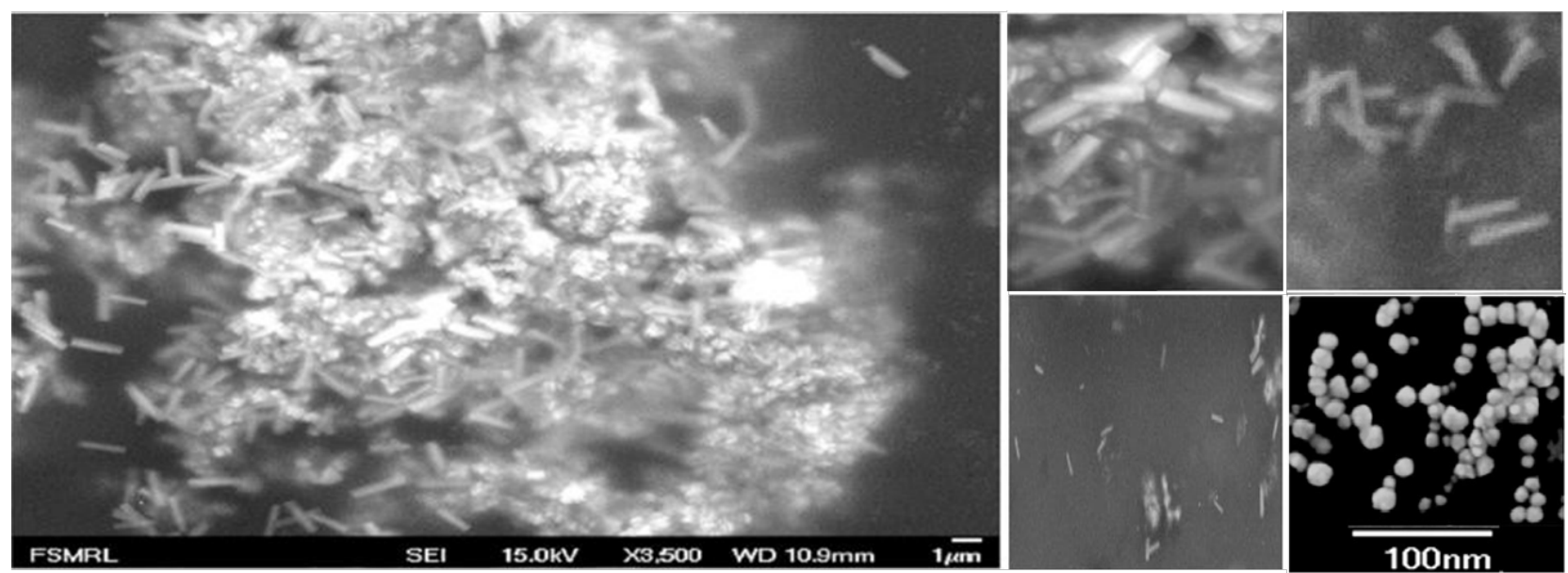

Figure 3. SEM images of GNRs and spherical particles (in presence of an emulsifier)
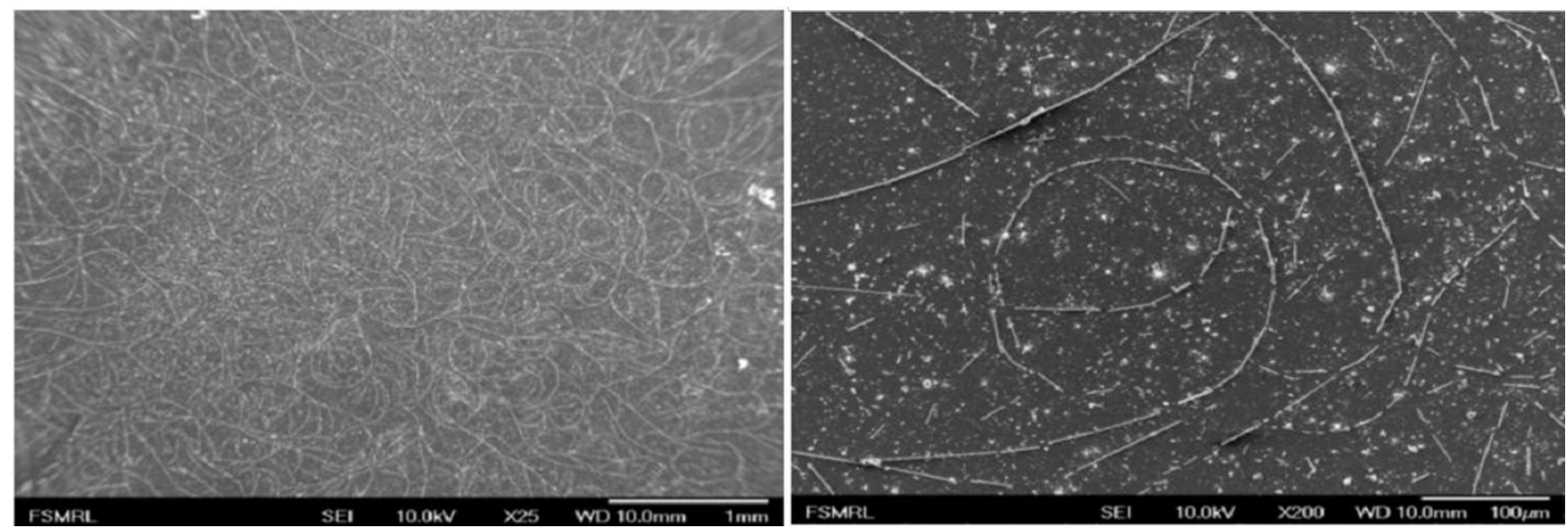

Figure 4. SEM images of gold nanofilaments in (absence of emulsifier)

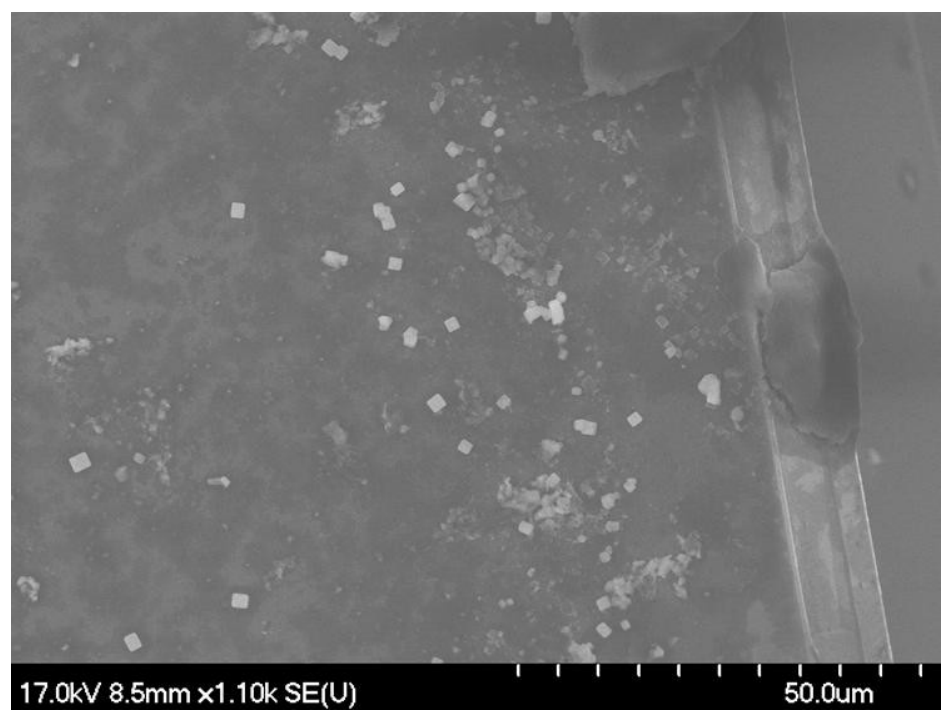

Figure 5. Gold nano plates in absence of emulsifier 
Figure 6A shows that nanocomponents beneath some of the plates/sheets are observable with SEM imaging (electronically visible) indicating that those sheets are ultrathin. To get a measurable signal from beneath, incident electrons must suffer absorption due to two passes through the top plate and a backscattering from the structure under. Using the relation $\mathrm{I}=\mathrm{I}_{0} \mathrm{e}^{-\mathrm{x} / \lambda}$ where $\mathrm{I}_{0}$ is the primary electron current, $\mathrm{I}$ is the transmitted current without deflections, $\mathrm{x}$ is the film thickness, and $\lambda$ is the mean free path. For a free mean path of $\lambda=1 \mathrm{~nm}$, and backscattering efficiency of 0.5 at $30 \mathrm{KeV}^{[33]}$, we get 0.07 for $\mathrm{I} / \mathrm{I} 0$ which is measurable.

Figure 6B shows several images of 2-D plates or sheets of gold in the act of side-to side (lateral) coalescence. The process is characterized by a growing neck. Observing coalescence at room temperature is consistent with the sheets being ultrathin ( $\sim 1 \mathrm{~nm}$ thick). In detailed simulations using a number of mechanisms models, it was found that coalescence between two identical particles accelerates for smaller particles since the melting temperature drops considerably for the infinitesimal particles $\left(\sim 300{ }^{\circ} \mathrm{C}\right.$ for $2.5-\mathrm{nm}$ nanoparticles, compared to $1064{ }^{\circ} \mathrm{C}$ for bulk) ${ }^{[34]}$.

Figure 6C shows several examples of when sheets are stacked on top of each other. In a complete contact configuration, vertical coalescence over the entire sheet takes place simultaneously. In this case sheets dissolve and fade away.
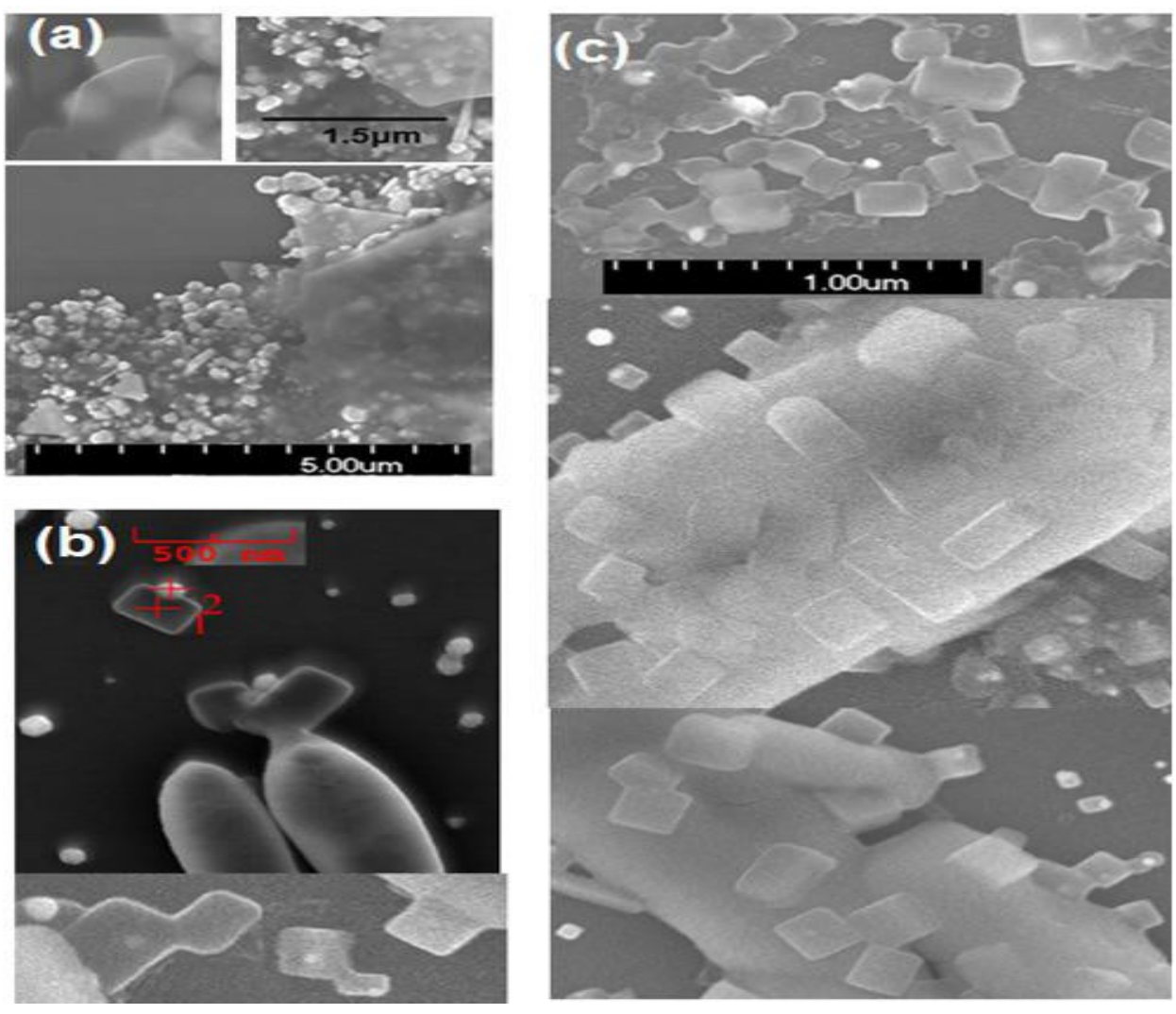

Figure 6. Vertical and lateral coalescence and melting of 2-D plates into each other (in the absence of emulsifier)

Figure 7 shows the elemental analysis by energy-dispersive spectrometry (EDS) taken with incident $30 \mathrm{KV}$ X-ray. It shows 2 characteristic peaks for gold at $2.13 \mathrm{eV}$ which given off as electrons return to the inner electron Ma shells of gold and a smaller peak at $9.7 \mathrm{eV}$ which confirms the presence of gold nanoparticles.

\subsubsection{Optical Microscope}

We used an optical microscope (magnification is $10 \times$ ) to observe the optical characteristics of the formations observed by SEM. Figure 8 gives two images of the sample and the left one is a close-up view for orange spots. Figure 8 shows the large assemblies of the gold filaments as dark brown. We also observe orange-green spots which are surrounded by light brown and dark brown spots. For regions where the green to yellow color is most dominant, we believe it is scattering from gold nanospheres ${ }^{[35]}$, corresponding to the surface plasmonic enhancement of scattering light in the 
visible region. This could also emanate from the plasomic transverse mode of nanorods. On the other hand, the dark and light brown areas are due to gold nanorods, corresponding to the surface plasmonic enhancement of the longitudinal oscillation.

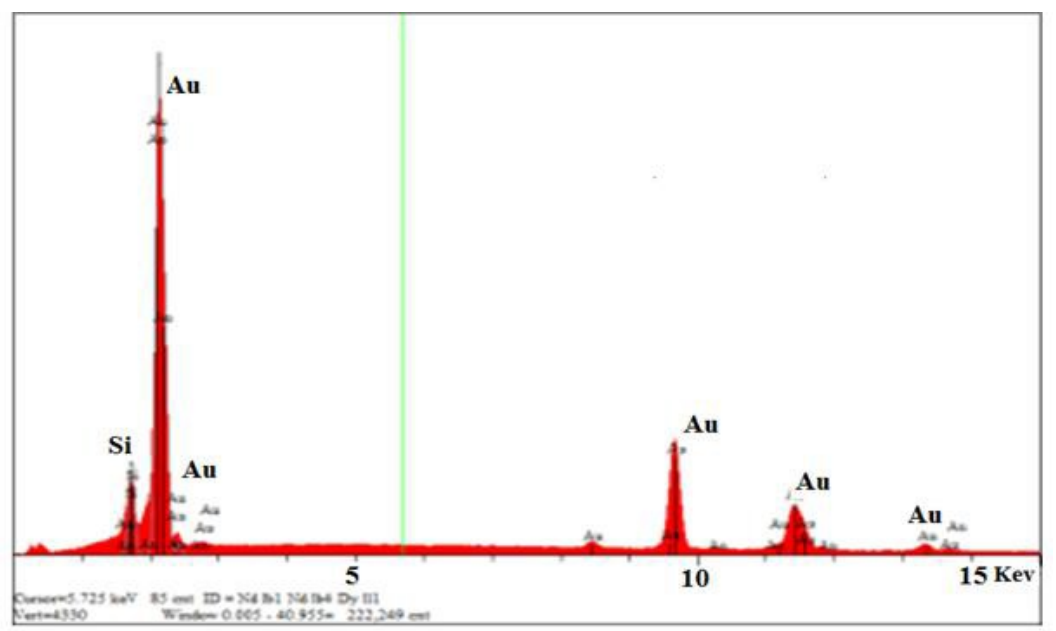

Figure 7. Energy dispersive spectrum for GNRs

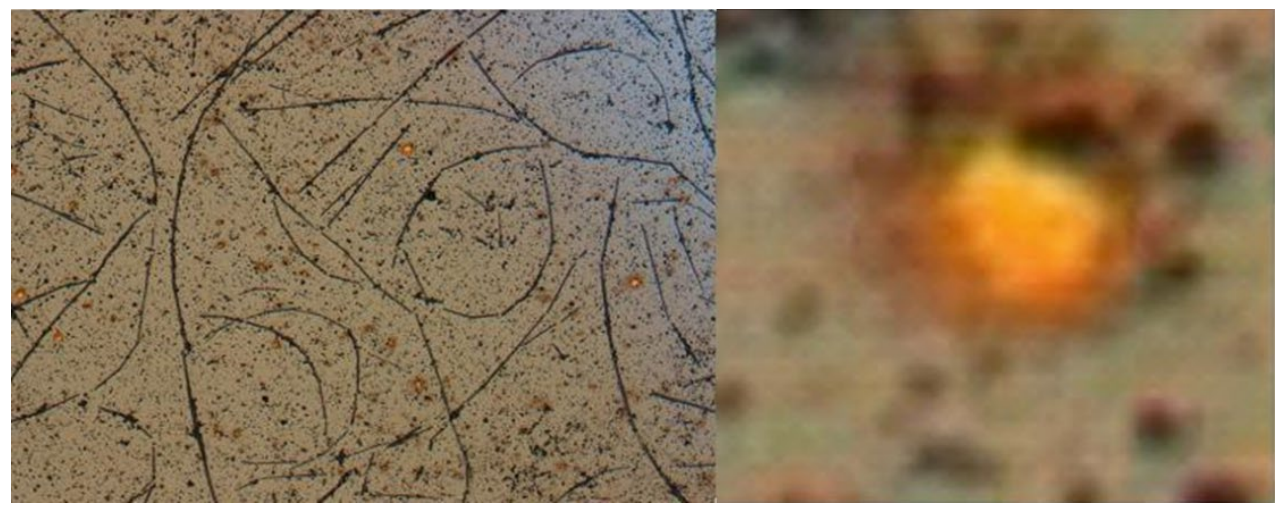

Figure 8. Light microscope images for GNRs and GNSs

\subsubsection{Photoluminescence measurements}

We have conducted the absorption measurements of the colloidal dispersion of the formed GNRs as well as $\mathrm{Si}$ nanoparticles dispersion in IPA under the UV-excitation at 365 using UV lamp. Figure 9a, b shows the luminescence spectra of SiNPs and GNRs respectively. As shown from figure 9a upon irradiation by Uv radiation at $365 \mathrm{~nm}$ the SiNPs emit orange red light in the range from 580 to $620 \mathrm{~nm}^{[36]}$. Figure $9 \mathrm{~B}$ shows that the red luminescence of the SiNPs disappeared as the gold nanostructure formed.

\section{Conclusion}

The present procedure represents the first demonstration of the use of semiconductor silicon nanoparticles seed to grow gold nano-rods without metal-based reducers or organic dimensional growth agents. It can also produce gold nanoplates and spheres. We recorded also millimeter-long filaments that exhibit anticrossing, as well as disordered end-to-end and end-to-side networks of gold nanorods. The resulting colloid was characterized. Our results show formation of gold nanorods with near uniform length of $1.5 \mu \mathrm{m}$ and diameter of $300 \mathrm{~nm}$ ( 5 aspect ratio). The goldsilicon (Au-Si) binary system belongs to the eutectic-alloy compounds, which exhibits lower melting temperatures and transform directly between solid and liquid. Our results showed that the formed sheets are stacked on top of each other and in this case the sheets dissolve and fade away. This will enable wafer-bonding, device connection and growth of gold nanorods and filaments using Si nanoparticles seeds. 


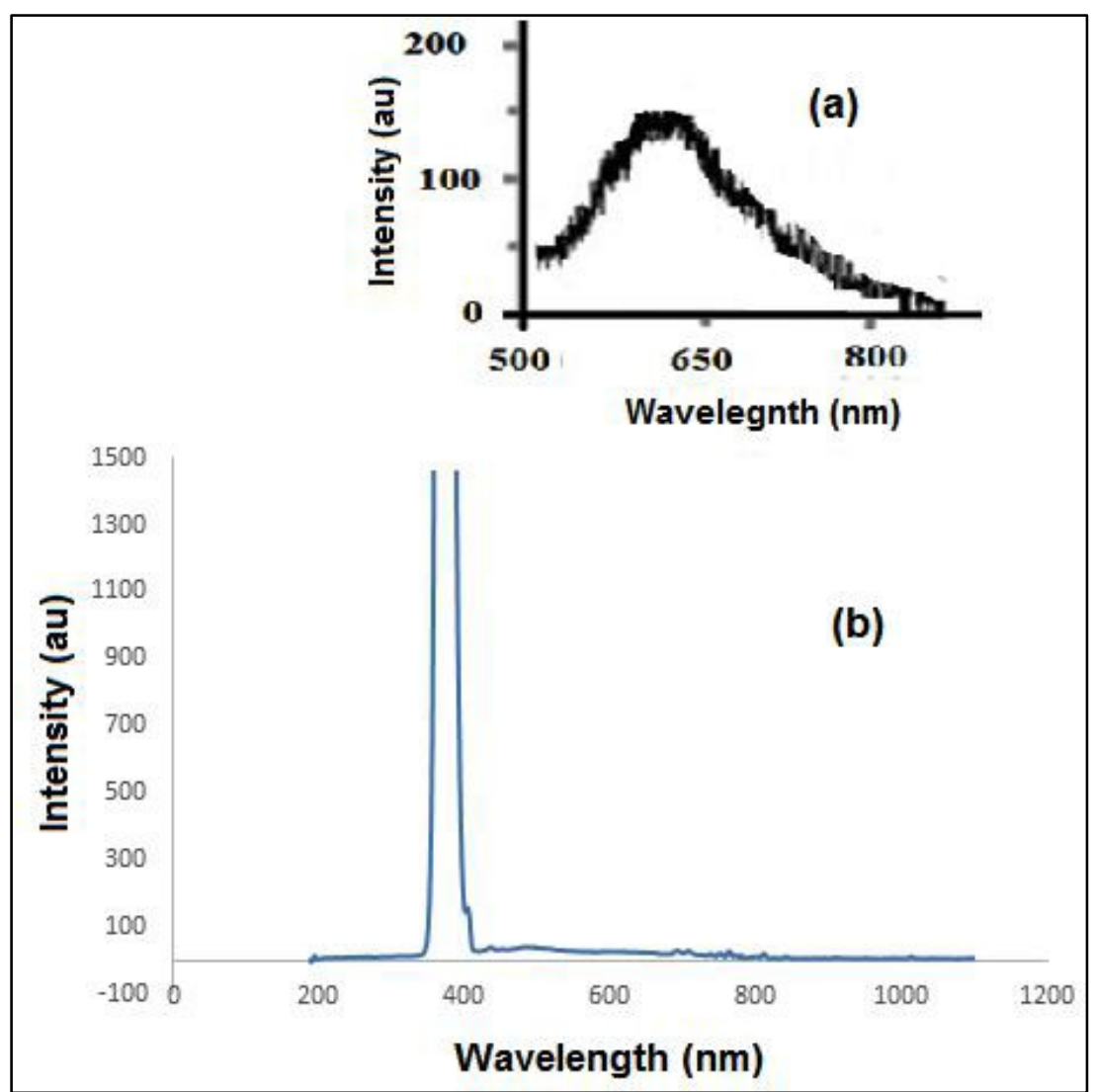

Figure 9. Photoluminescence spectra of (a) SiNPs and (b) GNRs.

\section{Acknowledgement}

The authors wish to thank the University of Illinois at Urbana Champaign (UIUC), USA, for generous assistance toward carrying out the necessary investigations in this work.

\section{References}

1. Daniel M C, Astruc A. Gold nanoparticles: assembly, supramolecular chemistry, quantum-size-related properties, and applications toward biology, catalysis, and nanotechnology. Chemical Reviews, 2004, 35(16): 293-346.

2. Glomm W R. Functionalized gold nanoparticles for applications in bio-nanotechnology. Journal of Dispersion Science \& Technology, 2005, 26(3): 389-414.

3. Becker R, Liedberg B, Käll P O. CTAB promoted synthesis of Au nanorods-temperature effects and stability considerations. Journal of Colloid \& Interface Science, 2010, 343(1): 25-30.

4. Pérez-Juste J, Pastoriza-Santos I, Liz-Marzán L M, et al. Gold nanorods: Synthesis, characterization and applications. Coordination Chemistry Reviews, 2005, 249(17): 1870-1901.

5. Murphy C J, Thompson L B, Chernak D J, et al. Gold nanorod crystal growth: from seed mediated synthesis to nanoscale sculpting. Current Opinion in Colloid \& Interface Science, 2011, 16(2): 128-134.

6. Ye X, Jin L, Caglayan H, et al. Improved size-tunable synthesis of nanodisperse gold nanorods through the use of aromatic additives. ACS Nano, 2012, 6(3): 2804-2817.

7. Huang X, Neretina S, El-Sayed M A. Gold nanorods: From synthesis and properties to biological and biomedical applications. Advanced Materials, 2009, 21(48): 4880-4910.

8. Yang D P, Cui D X. Advances and prospects of gold nanorods. Chemistry, An Asian Journal, 2010, 3(2): 20102022.

9. Vigderman L, Khanal B P, Zubarev E R. Functional gold nanorods: Synthesis, self-assembly and sensing applications. Advanced Materials, 2015, 24(36): 4811-4841.

10. Kennedy L C, Bickford L R, Lewinski N A, et al. A new era for cancer treatment: Gold-nanoparticle-mediated thermal therapies. Small, 2011, 7(2): 169-183.

11. Alkilany A M, Thompson L B, Boulos S P, et al. Gold nanorods: Their potential for photothermal therapeutics and drug delivery, tempered by the complexity of their biological interactions. Advanced Drug Delivery Reviews, 2012, 64(2): 190-199.

12. Martin C R. Membrane-based synthesis of nanomaterials. Chemistry of Materials, 1996, 8(8) 1739-1746.

13. Yu Y Y, Chang S S, Lee C L, et al. Gold nanorods: Electrochemical synthesis and optical properties. The Journal of Physical Chemistry B, 1997, 101(34): 6661. 
14. Niidome Y, Nishioka K, Kawasaki H, et al. Rapid synthesis of gold nanorods by the combination of chemical reduction and photoirradiation processes; morphological changes depending on the growing processes. Chemical Communications, 2003, 9(18): 2376-2377.

15. Kim F, Song J H, Yang P. Photochemical synthesis of gold nanorods. Journal of the American Chemical Society, 2002, 124(48): 14316-14317.

16. Okitsu K, Sharyo K, Nishimura R. One pot synthesis og gold nanorods by ultrasonic irradiation: The effect of $\mathrm{pH}$ on the shape of the gold nanorods and particles. Langmuir, 2009, 25: 7786-7790.

17. Biswal J, Ramnani S P, Tewari R, et al. Short aspect ratio gold nanorods prepared using gamma radiation in the presence of cetyltrimethyl ammonium bromide (CTAB) as a directing agent. Radiation Physics \& Chemistry, 2010, 79(4): 441-445.

18. Abidi W, Selvakannan P R, Guillet Y, et al. One-pot radiolytic synthesis of gold nanorods and their optical properties. Journal of Physical Chemistry C, 2010, 114(35): 14794-14803.

19. Obare S O, Jana N R, Murphy C J. Preparation of polystyrene- and silica-coated gold nanorods and their use as templates for the synthesis of hollow nanotubes. Nano Letters, 2001, 1(11): 601-603.

20. Sau T K, Murphy C J. Self-assembly patterns formed upon solvent evaporation of aqueous cetyl-trimethylammonium bromide-coated gold nanoparticles of various shapes. Langmuir, 2005, 21(7): 2923-2929.

21. Cheng J, Ge L, Xing B, et al. Investigation of $\mathrm{pH}$ Effect on Gold Nanorod Synthesis. Journal of the Chinese Chemical Society, 2011, 58(6): 822-827.

22. Ali M R K, Snyder B, El-Sayed M A. Synthesis and optical properties of small au nanorods using a seedless growth technique. Langmuir, 2012, 28(25): 9807-9815.

23. Scarabelli L, Grzelczak M, Liz-Marzán M L. Tuning gold nanorod synthesis through prereduction with salicylic acid. Chem. Mater, 2013, 25(21): 4232-4238.

24. Vigderman L, Zubarev E R. High-yield synthesis of gold nanorods with longitudinal SPR peak greater than 1200 $\mathrm{nm}$ using hydroquinone as a reducing agent. Chemistry of Materials, 2013, 25(8): 1450-1457.

25. Kang S K, Kim Y, Hahn M S, et al. Aspect ratio control of Au nanorods via temperature and hydroxylamine concentration of reaction medium. Current Applied Physics, 2006, 6(1): e114-e120.

26. Ma X, Wang M C, Feng J, et al. Effect of solution volume covariation on the growth mechanism of Au nanorods using seed-mediated method. Acta Materialia, 2005, 85: 322-330.

27. Pe'rez-Juste J, Pastoriza-Santos I, Liz-Marza'n L M, et al. Gold nanorods: Synthesis, characterization and applications. Coordination Chemistry Reviews, 2005, 249(17): 1870.

28. Okitsu K, Nunota Y. One pot synthesis of gold nanorods via autocatalytic growth of sonochemically formed gold seeds: The effect of irradiation time on the formation of seeds and nanorods. Ultrasonics Sonochemistry, 2014, 21(6): 1928-1932.

29. Nielsen D, Abuhassan L, Alchihabi M, et al. Current-less anodization of intrinsic silicon powder grains: Formation of fluorescent Si nanoparticles. Journal of Applied Physics, 2007, 101(11): 114302.

30. Rogozhina E, Belomoin G, Smith A, et al. Si-N linkage in ultrabright, ultrasmall Si nanoparticles. Applied Physics Letters, 2001, 78(23): 3711.

31. Rao S, Sutin J, Clegg R, et al. Excited states of tetrahedral single-core Si29 nanoparticles. The Physical Review, 2004, 69(20): 205319.

32. Abouie M, Liu Q, Ivey D G. Eutectic and solid-state wafer bonding of silicon with gold. Materials Science and Engineering B, 2012, 177(20): 1748- 1758.

33. Nguyen-Truong H T. Analytical formula for high-energy electron inelastic mean free path. Journal of Physical Chemistry C, 2015, 119: 23627.

34. Arcidiacono S, Bieri N R, Poulikakos D, et al. On the coalescence of gold nanoparticles. International Journal of Multiphase Flow, 2004, 30(7): 979.

35. Wang J X, Nilsson A M, Fernandes D L A, et al. Angle dependent light scattering by gold nanospheres. Journal of Physics: Conference Series, 2016, 682: 012018

36. Hoang T, Stupca M, Mantey K, et al. Complex of heavy magnetic ions and luminescent silicon nanoparticles. Journal of Applied Physics, 2013, 114(16): 164319. 